tein kinases differentially regulate eosinophilactivating cytokine release from human airway smooth muscle. Am. J. Respir. Crit. Care Med. 164:688-697.

18. Roth, M., et al. 2002. Interaction between glucocorticoids and beta2 agonists on bronchial airway smooth muscle cells through synchronised cellular signalling. Lancet. 360:1293-1299.

19. Pang, L., and Knox, A.J. 2001. Regulation of TNFalpha-induced eotaxin release from cultured human airway smooth muscle cells by beta2-agonists and corticosteroids. FASEB J. 15:261-269.
20. Mak, J.C., Hisada, T., Salmon, M., Barnes, P.J., and Chung, K.F. 2002. Glucocorticoids reverse IL-1beta-induced impairment of beta-adrenoceptor-mediated relaxation and up-regulation of G-protein-coupled receptor kinases. $\mathrm{Br} . \mathrm{J}$. Pharmacol. 135:987-996.

\section{Thyroid hormone action: a binding contract}

\author{
Mitchell A. Lazar
}

Division of Endocrinology, Diabetes, and Metabolism, Departments of Medicine and Genetics, and The Penn Diabetes Center, University of Pennsylvania School of Medicine, Philadelphia, Pennsylvania, USA

Thyroid hormones are critical for differentiation, growth, and metabolism. A new study (see the related article beginning on page 588) investigating the biological role of the TH receptor TR- $\beta$ has demonstrated that DNA binding is critical for most of its functions, but also suggests that novel mechanisms independent of DNA binding may contribute to regulation of auditory function by TR- $\beta$.

J. Clin. Invest. 112:497-499 (2003). doi:10.1172/JCI200319479.
Thyroid hormone $(\mathrm{TH})$ plays a critical role in the development and adult functions of many organs and tissues. Many of the effects of TH are mediated by a family of high-affinity receptor proteins, called $\mathrm{TH}$ receptors (TRs). Three functional TRs, TR- $\beta 1$, TR- $\beta 2$, and TR- $\alpha 1$, are encoded on two mammalian genes (1). The TR- $\alpha$ gene also encodes variant proteins that do not bind $\mathrm{TH}$ and whose function may be to inhibit the action of other TRs (2). The TRs are members of a larger family of nuclear receptors (NRs) for lipophilic signaling molecules that includes steroid hormones, vitamin derivatives such as retinoic acid and vitamin $\mathrm{D}_{3}$, fatty acid and cholesterol

\footnotetext{
Address correspondence to: Mitchell A. Lazar, University of Pennsylvania School of Medicine, 611 Clinical Research Building, 415 Curie Boulevard, Philadelphia, Pennsylvania 19104-6149, USA. Phone: (215) 898-0198; Fax: (215) 898-5408; E-mail: lazar@mail.med.upenn.edu. Conflict of interest: The author has declared that no conflict of interest exists.

Nonstandard abbreviations used: thyroid hormone (TH); $\mathrm{TH}$ receptor (TR); nuclear receptor (NR); retinoid X receptor (RXR); thyroid hormone response element (TRE); NR corepressor (N-CoR); silencing mediator of retinoid and thyroid receptors (SMRT); histone deacetylase (HDAC); thyroid-stimulating hormone (TSH); negative TRE (nTRE).
}

metabolites, and xenobiotics (3). As the family name suggests, a key aspect of TR function involves nuclear regulation of gene transcription. In this issue of the JCI, Shibusawa and colleagues use mice whose TR- $\beta$ gene products cannot bind DNA in order to shed light on the mechanisms by which TR functions in development and physiology (4). The TR- $\beta$ mutant mice have a phenotype that is similar but not identical to that of mice lacking TR- $\beta$, suggesting that most but not all TR functions involve direct DNA binding.

\section{Positive regulation of gene expression by $\mathrm{TH}$ : a simple model}

Like nearly all NRs, the central portion of TR contains a zinc-ordered domain that binds to double-stranded DNA

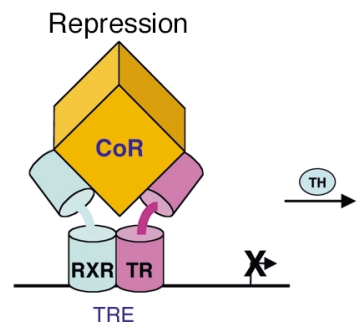

with a well-characterized sequence specificity. TR recognizes the sequence AGGTCA, to which it can bind as a monomer. This sequence also serves as a half-site for TR homodimers as well as heterodimers with the retinoid $X$ receptor (RXR) (5). RXR increases the DNA-binding affinity of TR and also restricts binding to a subset of half-site arrangements whose binding by the TR/RXR heterodimer is energetically favorable. The most stable binding occurs on the classical DR4 thyroid response element (TRE), in which two halfsites are directly repeated with a spacing of $4 \mathrm{bp}$ (6). The structural basis of this binding preference has been determined (7).

On a TRE-containing target gene, $\mathrm{TH}$ binding acts as a switch between repressed and activated states (Figure 1) (8). Genes that are bound by TR/RXR heterodimers, and potentially TR homodimers, are actively repressed in the absence of $\mathrm{TH}$. The repression function is located in the C-terminal ligand-binding domain, which binds to the corepressor molecules nuclear receptor corepressor (N-CoR) and silencing mediator of retinoid and thyroid receptors (SMRT) (9), which anchor large multiprotein complexes containing histone deacetylase (HDAC) activity and mediate ligand-independent repression. $\mathrm{TH}$ binding induces a conformational change that destabilizes corepressor binding and that favors binding of transcriptional coactiva-

.


a

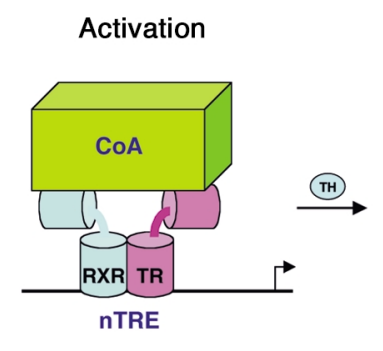

C Activation

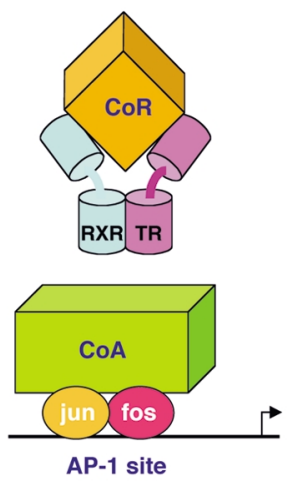

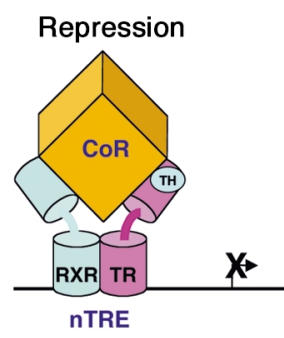

Repression
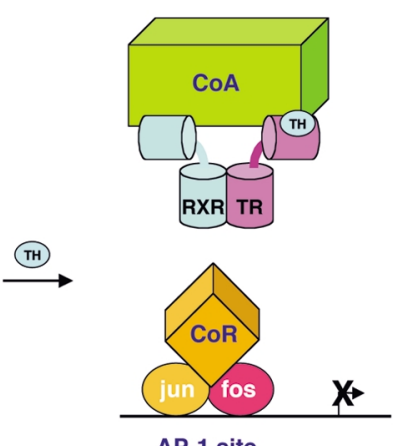

AP-1 site b Activation
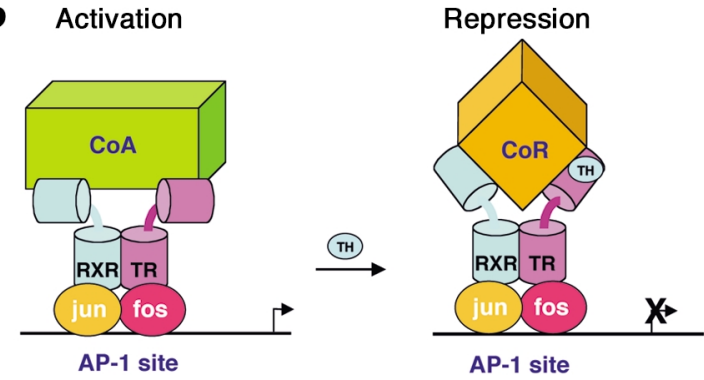

Figure 2

Potential mechanisms of negative regulation of gene expression by TH. (a) TH binding to TR triggers a switch from coactivator to corepressor binding on an nTRE on DNA. This mechanism absolutely requires direct DNA binding by TR. (b) TH binding to TR triggers a switch from coactivator to corepressor binding in the context of a protein-protein interaction, shown here with the AP-1 complex (jun/fos). (c) TH binding to TR recruits coactivator away from a DNA-bound factor such as AP-1.

tors, several of which possess histone acetyltransferase activity (10). This well-understood mechanism by which TH positively regulates gene expression would be expected to be completely abolished in the mutant mouse generated by Shibusawa et al. (4).

\section{Negative regulation of gene expression by $\mathrm{TH}$ : not so simple}

However, while positive regulation of gene expression by $\mathrm{TH}$ is nicely explained by this model, TH also downregulates numerous genes. Indeed, arguably the most important physiological effect of $\mathrm{TH}$ is negative regulation of thyrotropin (TSH) gene expression in the pituitary, which is critical to the normal function of the hypothalamic-pituitary-thyroid axis. The mechanism of negative regulation is not well understood, and several fundamentally different models have been postulated. In one model, the TR binds directly, via its DNA-binding domain, to a "negative" TRE (nTRE) in a negatively regulated gene. In prin- ciple, this could simply involve a reversal of the ligand-dependent switch between corepressor and coactivator binding (Figure 2a). Indeed, an nTRE has been identified in the TSH $\beta$ subunit gene (11), and TH-dependent recruitment of HDAC activity to the vicinity of this nTRE has been demonstrated (12). The mutant mouse defective in DNA binding by TR- $\beta$ described by Shibusawa et al. (4) provides the first test of this model in vivo, and the failure of TH to suppress TSH in these mice strongly supports the conclusion that negative regulation of the TSH gene by TH requires DNA binding by TR.

But what is the mechanism for this reversal of the switch paradigm? In vitro, TH clearly destabilizes the binding of corepressors N-CoR and SMRT and stabilizes the binding of coactivators. One possibility is that an nTRE-binding site might allosterically alter the conformation of TR such that corepressor binding is now favored in the presence of TH. Alternatively, a different set of coactivators and corepressors might be in- volved in ligand-dependent repression. The receptor-interacting protein RIP140 is an example of a cofactor that is recruited to ligand-bound TR and inhibits transcription (13). Binding of other transcription factors in the vicinity of the nTRE could also influence activity, as recently suggested for CTCF binding to the TR-dependent silencing element in the chicken lysozyme gene (14). The precise cofactors and downstream effectors of this form of DNA binding-dependent repression by $\mathrm{TH}$ remain to be determined.

There are at least three other mechanisms whereby $\mathrm{TH}$ could inhibit gene expression in a manner that does not involve the TR/DNAbinding domain and therefore would not be affected by a mutation that selectively impairs direct DNA binding by TR. One mechanism still involves recruitment of TR to the negatively regulated gene, but in this case the recruitment would be via a different DNA-binding protein, such as a component of AP- 1 complexes that have been demonstrated to interact with TR (15). In this model, 
the protein-protein interaction rather than the DNA might allosterically alter the conformation of ligandbound TR, leading to corepressor recruitment (Figure 2b) (16). Another mechanism for negative regulation is a squelching model, whereby TR acts in the nucleus to steal coactivators and corepressors (in the presence or absence of $\mathrm{TH}$, respectively) from other NRs as well as additional transcription factors (such as AP-1) that utilize the same coactivators or corepressors $(17,18)$ (Figure 2c). TR may also have nonnuclear effects, leading to activation of kinase cascades that ultimately impact on nuclear transcription factor function (19). The model described by Shibusawa et al. (4) should be of great value in determining which, if any, genes that are negatively regulated by TH use one of these mechanisms that does not require the intrinsic DNA-binding activity of TR. Unbiased microarray analysis of TH-target tissues might identify such genes in these mice.

\section{Implications for TH action in development}

Developmentally, the mutant mice defective in DNA binding by TR- $\beta$ exhibit a retinal defect that phenocopies that of the TR- $\beta$-null mouse (20). Although the target genes are not known, this complex phenotype undoubtedly involves both positive and negative regulation of gene expression by TR, and the present study indicates that DNA binding is necessary for the most important of these transcriptional effects. The TR- $\beta$-null mouse also exhibits profound neurosensory deafness (21). The mice whose TR- $\beta$ cannot bind DNA also have hearing defects, which likely result from abnormal regulation of genes to which TR- $\beta$ normally binds that are important for normal inner ear development and function. However, the auditory defect of the TR- $\beta$-null mouse phenotype is more severe than that of the DNA-binding mutant, suggesting that certain aspects of TR- $\beta$ function are independent of DNA binding. Understanding the residual functions of the mutant may provide insight into nonclassical functions of TR.

1. Lazar, M.A., and Chin, W.W. 1990. Nuclear thyroid hormone receptors. J. Clin. Invest. 86: $1777-1782$.

2. Flamant, F., and Samarut, J. 2003. Thyroid hormone receptors: lessons from knockout and knock-in mutant mice. Trends Endocrinol. Metab. 14:85-90.

3. Mangelsdorf, D.J., et al. 1995. The nuclear receptor superfamily: the second decazde. Cell. 83:835-839.

4. Shibusawa, N., et al. 2003. Thyroid hormone action in the absence of thyroid hormone receptor DNA-binding in vivo. J. Clin. Invest 112:588-597. doi:10.1172/JCI200318377.

5. Mangelsdorf, D.J., and Evans, R.M. 1995. The RXR heterodimers and orphan receptors. Cell. 83:841-850.

6. Umesono, K., Murakami, K.K., Thompson, C.C., and Evans, R.M. 1991. Direct repeats as selective response elements for the thyroid hormone, retinoic acid, and vitamin D3 receptors. Cell. 65:1255-1266.

7. Rastinejad, F., Perlmann, T., Evans, R.M., and Sigler, P.B. 1995. Structural determinants of nuclear receptor assembly on DNA direct repeats. Nature. 375:203-211.

8. Glass, C.K., and Rosenfeld, M.G. 2000. The coregulator exchange in transcriptional functions of nuclear receptors. Genes Dev. 14:121-141.

9. Hu, X., and Lazar, M.A. 2000. Transcriptional repression by nuclear hormone receptors. Trends Endocrinol. Metab. 11:6-10.

10. McKenna, N.J., Lanz, R.B., and O'Malley, B.W. 1999. Nuclear receptor coregulators: cellular and molecular biology. Endocr. Rev. 20:321-344.

11. Shibusawa, N., Hollenberg, A.N., and Wondisford, F.E. 2003. Thyroid hormone receptor DNA binding is required for both positive and negative gene regulation. J. Biol. Chem. 278:732-738.

12. Sasaki, S., et al. 1999. Ligand-induced recruitment of a histone deacetylase in the negativefeedback regulation of thyrotropin beta gene. EMBO J. 18:5389-5398.

13. Treuter, E., Albrektsen, T., Johansson, L., Leers, J., and Gustafsson, J.A. 1998. A regulatory role for RIP140 in nuclear receptor activation. Mol. Endocrinol. 12:864-881.

14. Lutz, M., et al. 2003. Thyroid hormone-regulated enhancer blocking: cooperation of CTCF and thyroid hormone receptor. $E M B O \mathrm{~J}$. 22:1579-1587.

15. Pfahl, M. 1993. Nuclear receptor/AP-1 interaction. Endocr. Rev. 14:651-658.

16. Rogatsky, I., Zarember, K.A., and Yamamoto, K.R. 2001. Factor recruitment and TIF2/ GRIP1 corepressor activity at a collagenase-3 response element that mediates regulation by phorbol esters and hormones. EMBO J. 20:6071-6083.

17. Kamei, Y., et al. 1996. A CBP integrator complex mediates transcriptional activation and AP-1 inhibition by nuclear receptors. Cell. 85:403-414.

18. Tagami, T., Madison, L.D., Nagaya, T., and Jameson, J.L. 1997. Nuclear receptor corepressors activate rather than suppress basal transcription of genes that are negatively regulated by thyroid hormone. Mol. Cell. Biol. 17:2642-2648.

19. Davis, P.J., Shih, A., Lin, H.Y., Martino, L.J., and Davis, F.B. 2000. Thyroxine promotes association of mitogen-activated protein kinase and nuclear thyroid hormone receptor (TR) and causes serine phosphorylation of TR. J. Biol. Chem. 275:38032-38039.

20. Ng, L., et al. 2001. A thyroid hormone receptor that is required for the development of green cone photoreceptors. Nat. Genet. 27:94-98.

21. Forrest, D., Erway, L.C., Ng, L., Altschuler, R., and Curran, T. 1996. Thyroid hormone receptor beta is essential for development of auditory function. Nat. Genet. 13:354-357. 